\title{
Continuous Arterial Spin Labeling of Mouse Cerebral Blood Flow Using an Actively-Detuned Two-Coil System at 9.4T
}

\author{
Hongxia Lei, Yves Pilloud, Arthur W. Magill and Rolf Gruetter
}

\begin{abstract}
Among numerous magnetic resonance imaging (MRI) techniques, perfusion MRI provides insight into the passage of blood through the brain's vascular network noninvasively. Studying disease models and transgenic mice would intrinsically help understanding the underlying brain functions, cerebrovascular disease and brain disorders. This study evaluates the feasibility of performing continuous arterial spin labeling (CASL) on all cranial arteries for mapping murine cerebral blood flow at 9.4T. We showed that with an active-detuned two-coil system, a labeling efficiency of $0.82 \pm 0.03$ was achieved with minimal magnetization transfer residuals in brain. The resulting cerebral blood flow of healthy mouse was $99 \pm 26 \mathrm{~mL} / 100 \mathrm{~g} / \mathrm{min}$, in excellent agreement with other techniques. In conclusion, high magnetic fields deliver high sensitivity and allowing not only CASL but also other MR techniques, i.e. ${ }^{1} \mathrm{H}$ MRS and diffusion MRI etc, in studying murine brains.
\end{abstract}

\section{INTRODUCTION}

$\mathrm{P}$ erfusion weighted imaging (PWI) is a powerful tool for studying brain vasculature, and the consequently derived cerebral blood flow (CBF) helps in assessing tissue viability and metabolic function. The increased number of mouse models allows studying brain functions and dysfunctions, such as hypoxia, stroke, tumor and neurodegeneration [1-3]. Thus measuring CBF in such disease models and genetically modified animals noninvasively would intrinsically help seeking potential biomarkers and assessing prospective treatments.

In MRI, arterial water molecules can be used as endogenous perfusion tracers. Two techniques, continuous arterial spin labeling (CASL) [4-8] and flow-sensitive

Manuscript received April $7^{\text {th }}, 2011$. This work was supported by Centre d'Imagerie BioMédicale (CIBM) of the UNIL, UNIGE, HUG, CHUV, EPFL and the Leenaards and Jeantet Foundations.

Hongxia Lei is with Laboratory of Functional and Metabolic Imaging, Ecole Polytechnique Federale de Lausanne, EPFL-SB-IPSB-LIFMET, CH F1 627, Station 6, Lausanne, CH1015, Switzerland (phone: +41-21-6937964; fax: +41-21-693-7960; e-mail: Hongxia.Lei@epfl.ch).

Yves Pilloud is with Laboratory of Functional and Metabolic Imaging, Ecole Polytechnique Federale de Lausanne, Lausanne, Switzerland (Yves.Pilloud@epfl.ch).

Arthur W. Magill is with Laboratory of Functional and Metabolic Imaging, Ecole Polytechnique Federale de Lausanne, Lausanne, Switzerland; Department of Radiology, University of Lausanne, Lausanne, Switzerland (Arthur.Magill@epfl.ch).

Rolf Gruetter is with Laboratory of Functional and Metabolic Imaging, Ecole Polytechnique Federale de Lausanne, Lausanne, Switzerland; Department of Radiology, University of Lausanne, Lausanne, Switzerland; Department of Radiology, University of Geneva, Geneva, Switzerland (Rolf.Gruetter@epfl.ch). alternating inversion recovery (FAIR) [9], are widely applied. In contrast to FAIR, CASL using an actively-detuned twocoil system presents higher sensitivity and minimizes magnetization transfer effects and thus is extensively used for studying rat brains [3-8]. However, non-invasive MR studies of $\mathrm{CBF}$ in mice using CASL remain challenging due to limiting factors such as reduced sensitivities because of the small volume of interest, amplified magnetic susceptibility and elevated physiological motion contributions. Thus the acquisition of high quality MR images of mouse brain with sufficient signal-to-noise ratios (SNRs) remains challenging. In addition, the distance between heart and brain in mouse is shorter than in that of rat, limiting CASL on carotid artery for CBF of mouse brain.

With increased magnetic fields, improved gradient performance, increased availability of high-order shim gradients and strengths of all shim gradients, and contemporary imaging techniques, imaging rodent brains at 9.4T and above using ultra-fast MR techniques, i.e. echoplanar imaging (EPI) has been shown to be feasible with satisfactory quality, i.e. minimal artifacts, less distortion and well-embedded anatomical structures [10].

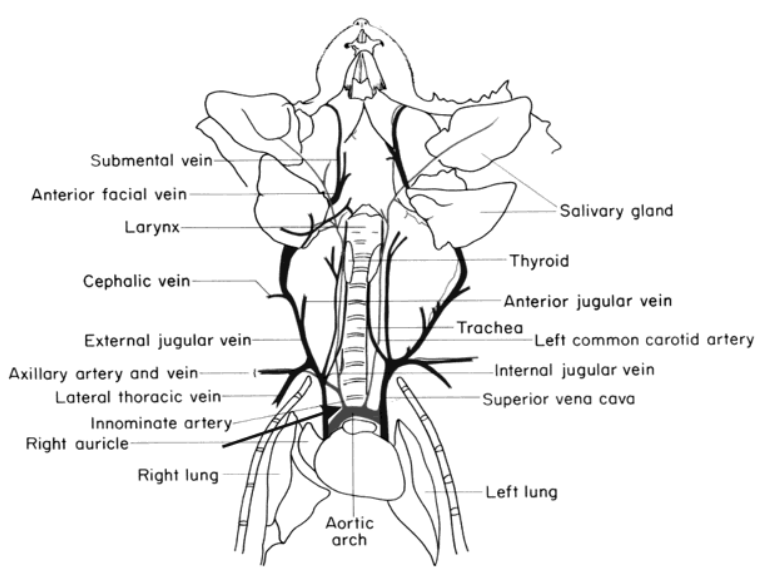

Fig. 1. Vessel maps of the mouse neck (http://www.informatics.jax.org/cookbook/subjectindex.shtml). The solid black arrow indicates a possible labeling plane right after the aortic arch when applying a z-gradient.

We noticed that all major intracranial arteries, i.e. both carotid arteries and the innominate artery (Fig.1), are located in front of the aorta artery towards brain. Such distance should be enough to allow delivery of sufficient labeled spins 
with minimal saturation effects when CASL is applied. The aim of this study was to employ an actively-detuned two-coil system to evaluate the feasibility of performing CASL for mapping $\mathrm{CBF}$ of mouse at 9.4T.

\section{METHODS}

\section{A. MR Instruments}

All MR experiments were carried out in a horizontal, 9.4T/26cm magnet (Magnex Scientific Inc., UK), with a 12$\mathrm{cm}$-diameter gradient $(400 \mathrm{mT} / \mathrm{m}$ in $200 \mu \mathrm{s}$, Magnex Scientific Inc., UK), interfaced to a DirectDrive console (Vnmrj, Varian Inc., USA). Eddy currents were minimized to less than $0.01 \%$ using time dependent quantitative eddy current field mapping [11]. A quadrature coil consisting of two geometrically decoupled 12-mm-inner-diameter loops, tuned to $400 \mathrm{MHz}$, was used as a radio-frequency (RF) transceiver for MR imaging. An 8-mm-inner-diameter butterfly coil, also resonating at $400 \mathrm{MHz}$, was used for labeling. Both coils include PIN-diode based active detuning circuits [12], and were controlled by the CASL pulse sequence via a home-built interface.

\section{B. CASL Pulse Sequence}

The CASL pulse sequence was implemented qw a semiadiabatic Spin-Echo EPI (SE-EPI) sequence with both negative and positive reference scans [10] by including additional labeling components, i.e. one 2.1 sec-long labeling RF pulse, a z-gradient (14 mT/m) and 1 second delay [4].

\section{Imaging Parameters}

Anatomical images of murine brain were acquired using fast spin echo (FSE) sequence $\left(\mathrm{TE}_{\text {effective }} / \mathrm{TR}=50 / 4000 \mathrm{~ms}\right.$, field of view $(F O V)=20 \mathrm{~mm} \times 20 \mathrm{~mm}$, readout $(\mathrm{RO}) \times$ phaseencode $(\mathrm{PE})=256 \times 256,8$ averages).

In order to illustrate the efficiency of the active-detuning system, sagittal images covering the entire neck region, were acquired with the labeling coil using a gradient echo (GRE) sequence $\quad(\mathrm{TE} / \mathrm{TR}=4 / 300 \mathrm{~ms}, \quad \mathrm{FOV}=25 \mathrm{~mm} \times 25 \mathrm{~mm}$, $\mathrm{RO} \times \mathrm{PE}=128 \times 128,8$ averages). Alternatively, coronal images of the neck region from the heart to the brain were acquired to locate the targeting cranial arteries.

First and second order shim terms were automatically adjusted over a $4 \mathrm{~mm} \times 6 \mathrm{~mm} \times 5 \mathrm{~mm}$ VOI using an EPI version of FASTMAP [13]. A localized water signal was acquired to examine the field homogeneity within the VOI.

Immediately after shimming, 16 pairs of 4-segmented semiadiabatic SE-EPI images were acquired to map blood flow $\left(\mathrm{TE}=40 \mathrm{~ms}, \quad \mathrm{FOV}=23 \times 15 \mathrm{~mm}^{2}, \quad \mathrm{RO} \times \mathrm{PE}=128 \times 64\right.$, spectral width $=200 \mathrm{kHz}, 0.8-\mathrm{mm}$ slice thickness). The total acquisition time was approximately 15 minutes.

\section{Animals}

All experiments were performed with approval from the local Institutional Animal Care and Use Committee. Six male C57/BL mice $(25-32 \mathrm{~g})$ were used for this study. Anesthesia was initially induced with $5 \%$ isoflurane, and maintained with $2 \%$ isoflurane well-mixed with oxygen.

Animals were stereotaxically fixed to a holder using ear bars and placed in the magnet. Throughout the experiment the rectal temperature of the animal was maintained at $\sim 36^{\circ} \mathrm{C}$ by circulating warm water. Breathing patterns and rectal temperature were simultaneously monitored using a MR-compatible monitoring system (Model 1025, SA Instruments Inc., USA). The breathing rate was maintained at $>100$ beats per minute by adjusting the rates of isoflurane in the range of $0.8-1.5 \%$, ensuring that $\mathrm{PaCO}_{2}$ of the arterial blood was in the range of $35-45 \mathrm{mmHg}$.

Two animals were sacrificed by overdosing isoflurane at the end of studies for assessing magnetization transfer effect of this experimental setting.

\section{E. Data Analysis}

CBF maps, in units of $\mathrm{mL} / 100 \mathrm{~g} / \mathrm{min}$, were derived by pair-wise pixel-by-pixel calculation of the paired images as previously described [4]:

$$
\mathrm{CBF}=\frac{\lambda}{T_{1}} \frac{S_{C}-S_{L}}{S_{L}+(2 \alpha-1) S_{C}}
$$

where $\lambda$ is the blood-brain partition coefficient for water ( 0.9 , [7]), $T_{1}$ is the brain tissue longitudinal relaxation time (1.9 sec at 9.4T, [7]), $\mathrm{S}_{\mathrm{C}}$ is the control imaging signal intensity, $\mathrm{S}_{\mathrm{L}}$ is the labeled image signal intensity, and $\alpha$ is the labeling efficiency of arterial spin labeling, assumed to be 0.8 [7].

Regions of interests (ROIs), such as frontal cortex, sensory-motor cortex, caudate putamen, thalamus, corpus callosum and hypothalamus, were hand drawn based on the acquired EPI images as described in [4].

\section{RESULTS}

When the two coils were placed around a phantom in a similar arrangement to that used for in vivo studies, coupling from either loop of the imaging coil to the tagging coil was measured to be less than $-23 \mathrm{~dB}$ using a network analyser (Model E5071C, Agilent Technologies Inc., USA), and further reduced by $27 \mathrm{~dB}$ using the active-detuning system. This was further confirmed by in vivo sagittal neck images (Fig. 2), in which almost no signal was observed when the imaging coil was detuned (Fig. 2B and 2D).

Multi-slice coronal images of the neck were obtained to ensure that the distance from the heart to the center of brain (Bregma 0) was $\sim 20 \mathrm{~mm}$. The major intracranial arteries of 
mice including the innominate artery and both carotid arteries were located $15-18 \mathrm{~mm}$ away from the center of brain. When the labeling pulse $(<1 \mathrm{~W})$ was applied along with the z-gradient component at the targeting plane, in front of the aortic arch towards brain and perpendicular to $\mathrm{B}_{0}$ (Fig. $1)$, the labeling efficiency was $82 \pm 3 \%$.
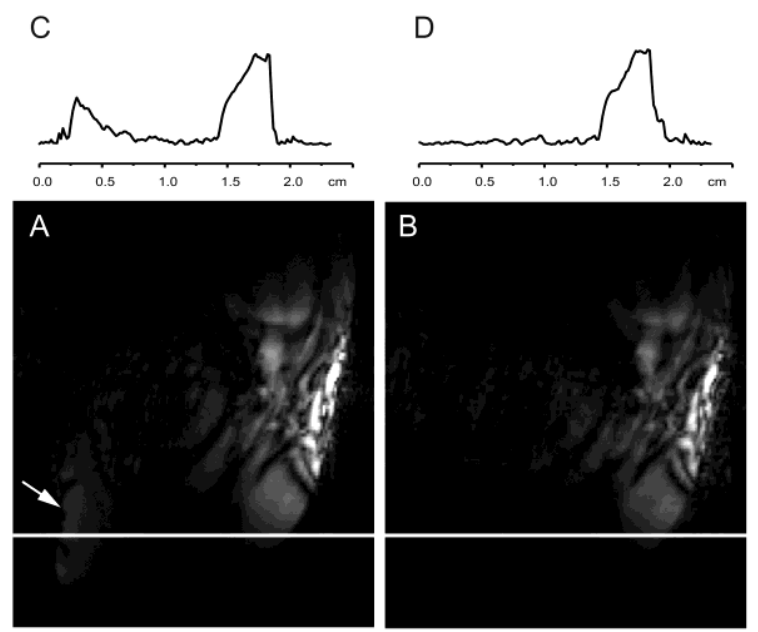

Fig. 2. Comparison of sagittal GRE images without/with active detuning. A and B are GRE images acquired using the neck coil as transceiver without and with active detuning image coil on top of the tagging coil. The arrow (in A) indicated the apparent signal intensity difference when comparing with $\mathrm{B}$. The corresponding project profiles indicated by lines in A and B were displayed in $\mathrm{C}$ and $\mathrm{D}$ respectively. In order to reach enough power generating such effect a large flip angle was applied at the neck coil position and thus resulted in distortions in the images $(\mathrm{A}, \mathrm{B})$.

After shimming, linewidths of the localized water signals were below $25 \mathrm{~Hz}$. SE-EPI images of mouse brain could be obtained with minimal artifacts and fine anatomical structures at 9.4T (Fig. 3). After the paired SE-EPI images were acquired, CBF maps were calculated according to Eq (1) and resulted in an average $\mathrm{CBF}$ over the entire brain of $99 \pm 26 \mathrm{~mL} / 100 \mathrm{~g} / \mathrm{min}$ (Fig. 3B, summarized in Table 1).

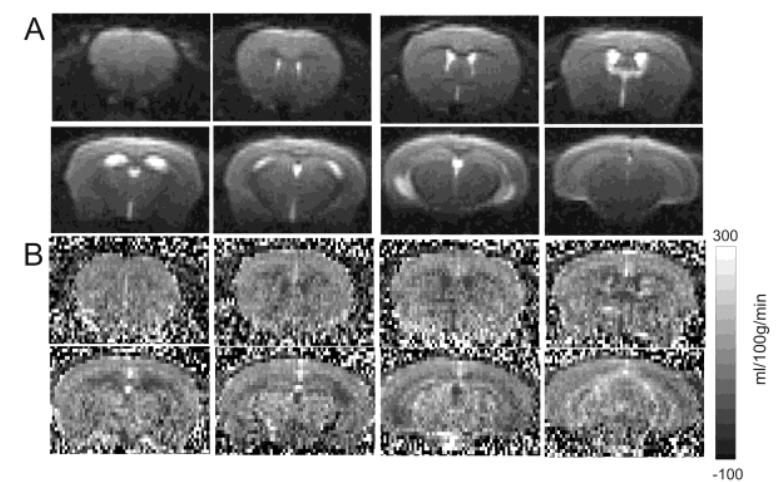

Fig. 3. Typical multi-slice 4-segemented semi-adiabatic SE-EPI images (A, TE/TR $=40 / 2000 \mathrm{~ms}$ ) and the derived CBF maps (B) of one mouse brain at $9.4 \mathrm{~T}$.

\section{DISCUSSION}

In our results, the measured labeling efficiency was very close to that in rats [7] but slightly higher than that from an ASL study labeling the blood in the heart [4]; the quantitative $\mathrm{CBF}$ results were comparable to that from previous cardiac-spin-labeling [4] and $\left[{ }^{14} \mathrm{C}\right]$-Iodoantipyrine results [14]. In addition, there was no observable magnetization transfer effects measured in the postmortem brains under identical experimental conditions. This suggests that applying spin labeling close to the edge of the aorta arch and towards brain can efficiently deliver sufficient labeled spins to the imaging planes with minimal magnetization transfer effects.

In present study, we applied a semi-adiabatic segmented SE-EPI sequence to measure CBF maps of murine brains using the surface coil (see in methods) and obtained the quality images (Fig. 3). At higher magnetic fields, i.e. 14.1T, the similar quality of rodent brain images remained to be preserved using the similar approaches $[10,15]$. On top of that, the slightly increased $T_{1}$ of blood water at higher magnetic field [16] will intrinsically minimize the effect of the transit time. Thus, CASL of mouse using the two-coil system should be possible at higher magnetic fields $(>9.4 \mathrm{~T})$ with improved sensitivity and therefore shortened the acquisition time. The particular EPI technique has been used for acquiring diffusion tensor images of rats [10] and mice at $14.1 \mathrm{~T}$ [15]. In addition, recent proton spectroscopy studies in murine brains at $9.4 \mathrm{~T}$ and above, suggested that higher sensitivity could be achieved with the surface coil when compared to a volume coil and thus allowed studying metabolites in brain regions after neurodegeneration or dysfuntions [17,18], such as evolution of neurochemical profile of striatal lesion from mouse brain after transient focal ischemia [19]. Thus, studying murine brain using various MRI and MRS methods is feasible at 9.4T or above.

TABLE 1

\begin{tabular}{lc}
\multicolumn{2}{c}{ SUMMARY OF REGIONAL CBF OF MOUSE } \\
\hline Region of interest (ROI) & $\begin{array}{c}\text { CBF } \\
(\mathrm{ml} / 100 \mathrm{~g} / \mathrm{min})\end{array}$ \\
\hline Frontal Cortex & $108.8 \pm 4.5$ \\
Sensory-Motor Cortex & $118.7 \pm 7.8$ \\
Caudate Putamen & $99.3 \pm 7.8$ \\
Thalamus & $125.6 \pm 20.8$ \\
Corpus Callosum & $53.2 \pm 2.3$ \\
Hypothalamus & $88.4 \pm 13.6$ \\
\hline $\begin{array}{l}\text { Summary of regional blood flow of mouse brain }(\mathrm{n}=6) . \text { The } \\
\text { data were displayed as mean } \pm \text { standard deviations. }\end{array}$
\end{tabular}

\section{CONCLUSION}

We conclude that, the CASL technique of murine brain 
using two actively-detuned surface coils can be implemented at high magnet fields, i.e. 9.4T. This offers the possibility of studying various transgenic mouse models with increased sensitivities by means of multi-MR techniques.

\section{ACKNOWLEDGMENT}

This study was supported by Centre d'Imagerie BioMédicale (CIBM) of the UNIL, UNIGE, HUG, CHUV, EPFL and the Leenaards and Jeantet Foundations.

\section{REFERENCES}

[1] J.R. Petrella, J.M. Provenzale, "MR perfusion imaging of the brain: techniques and applications," Am J Roentgenol 2000; 175:207-219;

[2] M. Wintermark, M. Sesay, E. Barbier, et al., "Comaprative overview of brain perfusion imaging techniques," Stroke $2005 ; 36: 2032$

[3] D.L. Thomas, "Arterial spin labeling in small animals: methods and applications to experimental cerebral ischemia," J Magn Reson Imaging 2005; 22:741-744

[4] E.R. Muir, Q. Shen, T.Q. Duong, "Cerebral blood flow MRI in mice using the cardiac-spin-labeling technique, ". Magn Res Med 2008; 60:744-748;

[5] D.S. Williams, J.A. Detre, J.S. Leigh, A.P. Koretsky, "Magnetic resoance imaging of perfusion using spin inversion of arterial water," Proc Natl Acad Sci (USA) 1992; 89:212216 ;

[6] W. Zhang, D.S. Williams, J.A. Detre, A.P. Koretsky, "Measurement of rat brain perfusion by NMR using spin labeling of arterial water: in vivo determination of the degree of spin labeling," Magn Res Med 1993; 29:416-421;

[7] I.Y. Choi, S.P. Lee, S.G. Kim, R. Gruetter, “ In vivo measurements of brain glucose transport using the reversible Michaelis-Menten model and simultaneous measurements of cerebral blood flow changes during hypoglycemia,". J Cereb Blood Flow Metab 2001; 21:653-663;

[8] X. Zhang, T. Nagaoka, E.J. Auerbach, R. Champion, L. Zhou, T.Q. Duong, " Quantitative basal CBF and CBF fMRI of rhesus monkeys using three-coil continuous arterial spin labeling,". NeuroImage 2007; 34:1074-1083;

[9] S.G. Kim, "Quantification of relative cerebral blood flow change by flow-sensitive alternating inversion recovery (FAIR) technique: application to functional mapping," Magn Res Med 1995; 34:293-301;

[10] Y. van de Looij, N. Kunz, P Huppi, R. Gruetter, S. Sizonenko, "Diffusion tensor echo planar imaging using surface coil transceiver with a semi-adiabatic RF pulse sequence at 14.1T," Magn Res Med 2011; 65:732-737;

[11] M. Terpstra , P.M. Andersen, R. Gruetter "Localized eddy current compensation using quantitative field mapping," $J$ Magn Reson. 1998; 131(1):139-43;

[12] P.M. Mellor and D.R. Checkley, "Active coil isolation in NMR imaging and spectroscopy using PIN diodes and tuned transmission line: a practical approach," $M A G M A$ 1995; 3:3540

[13] R. Gruetter “ Automatic, localized in vivo adjustment of all first- and second-order shim coils,"Magn Res Med 1993; 29:804-811;

[14] T.M. Jay, G. Lucignani, A.M. Crane, J. Jehle, L. Sokoloff, "Measurement of ocal cerebral blood flow with $\left[{ }^{14}\right.$ C]iodoantipyrine in the mouse,". J Cereb Blood Flow Metab 1988; 8:121-129;

[15] H. Lei, Y. Van de Looij Y, N. Kunz, C. Berthet, L. Hirt and R. Gruetter "Diffusion tensor echo planar imaging of mouse brain after brief focal middle cerebral artery occlusion at 14.1T," Proceedings $18^{\text {th }}$ Scientific Meeting of the International Society for Magnetic Resonance in Medicine, 2010; 2234;

[16] R.A. de Graaf, P.B. Brown, S. McIntyre, T.W. Nixon. K.L. Behar, D.L. Rothman "High magnetic field water and metabolite proton T1 and T2 relaxation in rat brain in vivo," Magn Res Med 2006; 56(2):386-394;

[17] I. Tkac, P.G. Henry, P. Andersen, C.D. Keene, W.C. Low, R. Gruetter "Highly resolved in vivo ${ }^{1} \mathrm{H}$ NMR spectroscopy of the mouse brain at 9.4T, " Magn Res Med 2004; 52(3):478484;

[18] H. Lei, C. Poitry-Yamate, F. Preitner, B. Thorens, R. Gruetter "Neurochemical profile of the mouse hypothalamus using in vivo ${ }^{1} \mathrm{H}$ MRS at 14T ," NMR in Biomed 2010 23(6):578-583;

[19] H. Lei, C. Berthet, L. Hirt, R. Gruetter " Evolution of the neurochemical profile after transient focal cerebral ischemia in the mouse brain, " J Cereb Blood Flow Metab 2009; 29(4):811-819 